\title{
Il francese tra intenzione e realtà: un approccio giuridico a partire da spunti problesociolinguistici
}

Nicolò Alessi

\section{(2) OpenEdition \\ Journals}

Edizione digitale

URL: https://journals.openedition.org/esp/4330

DOI: $10.4000 /$ esp.4330

ISSN: 2532-0319

Editore

Centre d'Information sur l'Éducation Bilingue et Plurilingue

\section{Edizione cartacea}

Data di pubblicazione: 1 décembre 2019

Paginazione: 5-15

ISSN: $1127-266 X$

Notizia bibliografica digitale

Nicolò Alessi, «ll francese tra intenzione e realtà: un approccio giuridico a partire da spunti problesociolinguistici», Éducation et sociétés plurilingues [Online], 47 | 2019, Messo online il 01 septembre 2021, consultato il 02 septembre 2021. URL: http://journals.openedition.org/esp/4330 ; DOI: https://doi.org/10.4000/esp.4330 


\title{
IL FRANGESE TRA INTENZIONE E REALTÀ: UN APPROGCIO GIURIDICO A PARTIRE DA SPUNTI PROBLESOCIOLINGUISTICI
}

\begin{abstract}
Nicolò ALESSI
Après une introduction retraçant l'origine de la présence du français en Vallée d'Aoste et son régime juridique, l'article compare les intentions qui ont justifié la protection du français dans le système juridique valdôtain à la réalité de son emploi effectif. De ce point de vue, le hiatus qui s'est formé entre la position juridique $d u$ français - basée sur son équivalence par rapport à l'italien, l'autre langue officielle - et celle que, dans la réalité, il a atteint apparaît. En effet, si on analyse avec réalisme ce que le français représente dans le domaine valdôtain, on aperçoit une langue peu pratiquée et avec laquelle la population valdôtaine a un rapport faussé pour plusieurs raisons, dont le rôle important de l'approche politique de la question linguistique. L'article propose, dans sa partie finale, des idées pour donner un nouvel élan au français en Vallée d'Aoste.
\end{abstract}

Mots-clés: français, Vallée d'Aoste, système juridique

After reviewing the origins of the presence of French in the Aosta Valley and its legal framework, the article compares the intentions that justified giving it legal protection to the reality of its actual use in the Region. From that point of view, there is a clear hiatus between the legal position of French-based on its equivalence with Italian, the other official language - and the position it has actually attained. In fact, if we consider language practices in the Valley realistically, French is scarcely practiced and the population's relationship with it is problematic, for several reasons, among which the crucial role of the political approach to the linguistic issue. In its last section, the article offers a ferw ideas to boost the French language anew in the Aosta Valley.

Keywords: French; Aosta Valley; legal system; minority linguistic rights; language policies

IL FRANCESE NELLE INTENZIONI 'ordinamento regionale speciale per la tutela della minoranza francofona è stato creato secondo il modello delle $\checkmark$ altre Regioni alpine di confine.

Tra le ragioni sottese alla creazione della Regione Valle d'Aosta, la questione linguistica ha ovviamente un ruolo preminente. Questo territorio ha uno storico legame con l'area linguistica francofona a partire dall'espansione su di esso della sfera d'influenza del Regno di Borgogna; un legame che va saldandosi con l'instaurazione e l'infittirsi dei peculiari rapporti col Ducato di Savoia. Nel territorio valdostano il francese si consolida come lingua colta e, soprattutto, come lingua della Chiesa, dell'amministrazione e della scuola. A essa si accompagna una diffusa pratica 


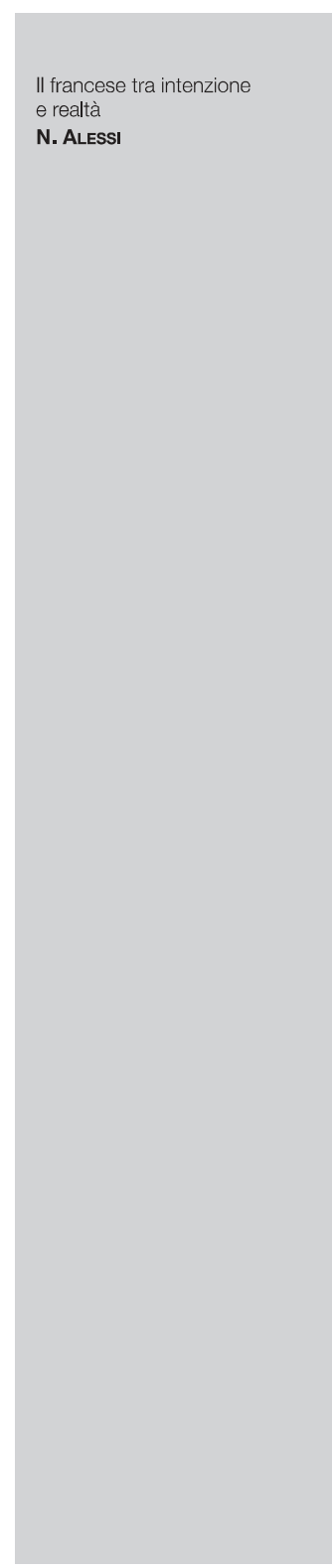

di parlate condivise con l'area francoprovenzale.

Senza dubbio le politiche liberali prima e fasciste poi hanno inciso rapidamente e profondamente sul tessuto sociolinguistico valdostano imponendo la diffusione dell'italiano come lingua della nuova nazione e, in seguito, del regime. La tendenza uniformatrice di questi due regimi politici ha incontrato le resistenze (di una parte) della popolazione valdostana, che, dopo la Liberazione, ha ottenuto una specifica protezione del proprio patrimonio linguistico-culturale. La prima soluzione adottata nel convulso secondo dopoguerra valdostano è il d.lgs.lgt. 7 settembre 1945, n. 545, che istituisce la Circoscrizione autonoma della Valle d'Aosta e, tra le altre cose, ristabilisce il libero uso della lingua francese.

$\mathrm{Ma}$ è in seno alla Costituente che viene data una soluzione stabile alla questione valdostana, seguendo un modello applicato anche per altri contesti regionali alpini di confine caratterizzati da peculiarità linguistiche. E in effetti allo Statuto speciale - e non all'art. 6 della Costituzione - che bisogna guardare per identificare la principale fonte di tutela dei diritti linguistici della popolazione valdostana (Palici di Suni 2002: 26, 29-30). La creazione di ordinamenti regionali speciali rappresenta invero la soluzione originariamente prescelta dal legislatore costituzionale per la tutela dei diritti linguistici dei gruppi alloglotti presenti sul territorio italiano. Definiti come minoranze linguistiche cosiddette nazionali ossia gruppi linguistici parlanti (anche) una lingua nazionale di uno Stato confinante - essi rappresentano, nell'ottica dei costituenti, gli unici possibili destinatari di misure di tutela, tanto da far caldeggiare in sede di Assemblea costituente un'eliminazione del più generale art. 6 , considerato ai tempi una protezione alternativa a questo modello.

Ovviamente la specialità valdostana non poggia solo sul dato linguistico, essendo, più in generale, le caratteristiche peculiari di un contesto morfologico, politico, culturale a essere messe in risalto con questo ordinamento speciale. Resta il fatto che la tutela della pratica linguistica rappresenta una delle ragioni fondamentali poste alla base della specialità valdostana. Dal punto di vista dei diritti linguistici, l'ordinamento regionale sembra rispondere a una ratio fondamentale, ossia quella di «restituire» alla popolazione valdostana i diritti goduti in passato, ripristinando la condizione vissuta dalla Regione prima delle politiche uniformatrici. 


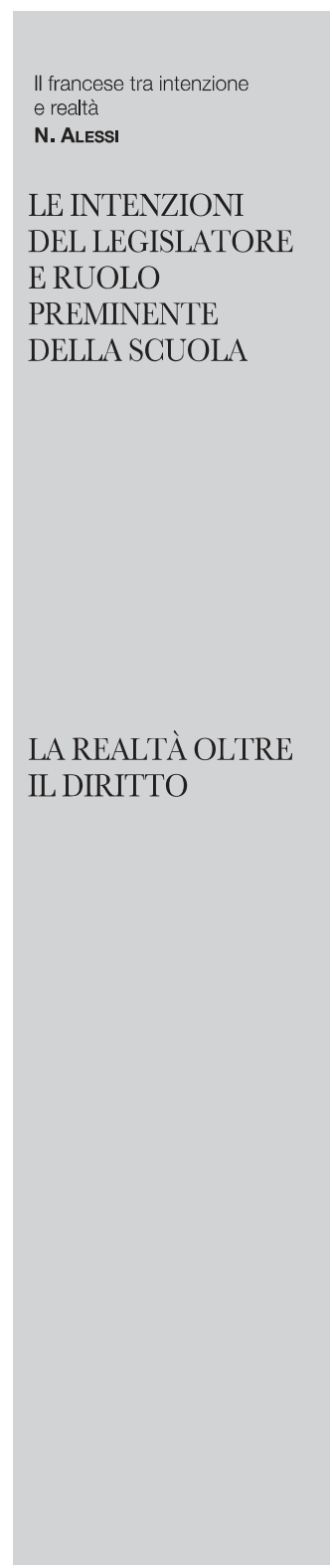

In questo senso, il legislatore costituzionale si pone gli obiettivi di restituire alla popolazione la facoltà di usare il francese - ristabilendo le condizioni per l'utilizzo di questa lingua - e di incoraggiarne la pratica. Lo Statuto speciale, in effetti, da una parte attribuisce pari dignità alle lingue italiana e francese promuovendo altresì il libero uso di quest'ultima nella redazione degli atti pubblici e dell'autorità giudiziaria (salvo le sentenze), e, dall'altra, dispone una strutturazione bilingue della scuola. Le norme rappresentano dunque la volontà di tutelare la lingua consentendone espressamente l'utilizzo nei settori ove essa era maggiormente d'uso anche in passato, strutturando un'amministrazione regionale bilingue e incoraggiando la promozione della lingua attraverso la scuola.

In sintesi, si può pertanto definire la disciplina statutaria del francese come «restitutoria/ripristinatoria» poiché volta a garantire nuovamente le condizioni di libero utilizzo del francese in Valle d'Aosta (a garanzia dei cosiddetti diritti linguistici primari), e «promozionale» in quanto diretta a incoraggiare la pratica del francese soprattutto attraverso la leva scolastica.

La scuola, la politica e le istituzioni determinano una settorializzazione del francese. Il dato formale della creazione di un ordinamento speciale ispirato a un'ottica «restitutoria/ripristinatoria»e «promozionale» sconta un confronto con diversi dati di realtà che ne relativizzano la portata.

In primo luogo, il più evidente: il fine ripristinatorio non ha riscontro in una realtà nella quale la popolazione valdostana, alla prova dei fatti, non può essere definita, in termini sociolinguistici, bilingue in senso stretto (1). In altre parole, è evidente che le previsioni statutarie abbiano stabilito un ordinamento che ha come destinataria una popolazione completamente diversa rispetto a quella che, ancora ai primi del Novecento, parlava diffusamente il francese, quantomeno in alcuni contesti. L'ordinamento «restitutorio/ripristinatorio», caratterizzato dalla garanzia formale della pari dignità tra le lingue, non trova riscontro in una realtà nella quale la lingua francesc è scarsamente utilizzata.

Anche il fine "promozionale» insito nello Statuto speciale, volto a quella che è stata definita una «rifrancesizzazione» dei valdostani attraverso la previsione di strumenti - soprattutto di politica scolastica - per incentivare l'utilizzo della lingua, non può dirsi raggiunto, e non era realisticamente raggiungibile. Semplificando, strutturare una scuola bilingue non basta a ricreare abitudini sociolinguistiche che sono la conseguenza di profonde trasforma- 


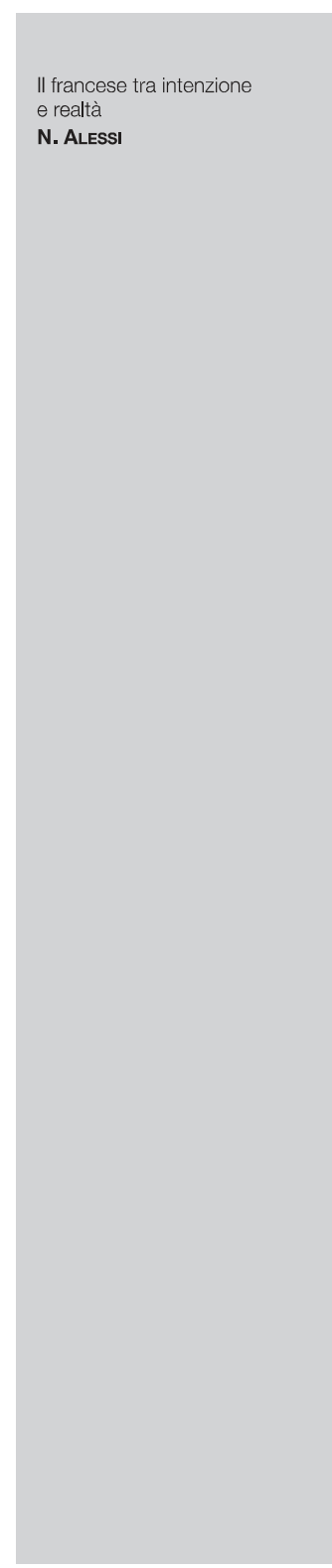

zioni del tessuto sociale valdostano.

Ciò che si vuole affermare è che la costruzione costituzionale di un ordinamento regionale speciale connotato da peculiarità linguistiche si scontra con una realtà diversa da quella che traspare dal dettato normativo e difficilmente realizzabile nel concreto. In altre parole, l'ordinamento regionale potrebbe essere definito, per certi versi, velleitario.

Possono essere identificati diversi fattori che hanno rafforzato un'impostazione non realistica della questione linguistica in Valle d'Aosta, scoraggiandone un ripensamento volto a restituirle un significato. Tra questi, sicuramente l'appropriazione politica dell'argomento della tutcla della lingua francese - che lega indissolubilmente la questione linguistica a un atteggiamento «difensivo» della comunità e dell'identità regionali - ha contribuito ad amplificarne il distacco dalla realtà. Essa ha rafforzato la sensazione che per il francese si tratti di un portato della tradizione - da rispettare perché fattore di distinzione e «innalzamento» della comunità valdostana rispetto alle altre (e, dunque, in definitiva, fattore di esclusione e non di arricchimento) - al quale riservare degli spazi, ma dai confini ben determinati. In questo modo il francese è stato inevitabilmente circoscritto in precise attività e precisi temi ricorrenti, con scarse opportunità di crescita e di affermazione nella società.

Si può dire, in effetti, che il francese sopravviva all'interno di «riserve» all'interno delle quali si percepisce una pratica artefatta e lontana dalla realtà. Così è nella scuola, ove il francese, salve le eccezioni delle scuole dell'infanzia e della scuola primaria, è in modo ancora preponderante lingua meramente insegnata e non d'insegnamento; ciò è ancora più vero se si considera l'attività politica e istituzionale, che legano l'uso del francese a precisi spazi «obbligati» e a determinate materie trattate, ossia quelle che hanno maggiormente a che fare con le specificità della Regione $\mathrm{e}$ che, generalmente, non presentano un contenuto eccessivamente complesso.

A tal proposito, un'indagine condotta sulla totalità delle leggi regionali conferma questa impostazione di fondo dell'attività istituzionale della Regione. Le leggi regionali approvate in francese rappresentano l'1,5\% della totalità delle leggi (48 su 3117), e le materie oggetto di disciplina sono la scuola, la cultura francofona (soprattutto sotto forma di contributi per associazioni o iniziative culturali per la francofonia), e, in parte minore, la toponomastica e il territorio (comprese, in alcuni casi, le attività agricole).

L'attività della Giunta regionale, analizzata nell'anno 2018, con- 


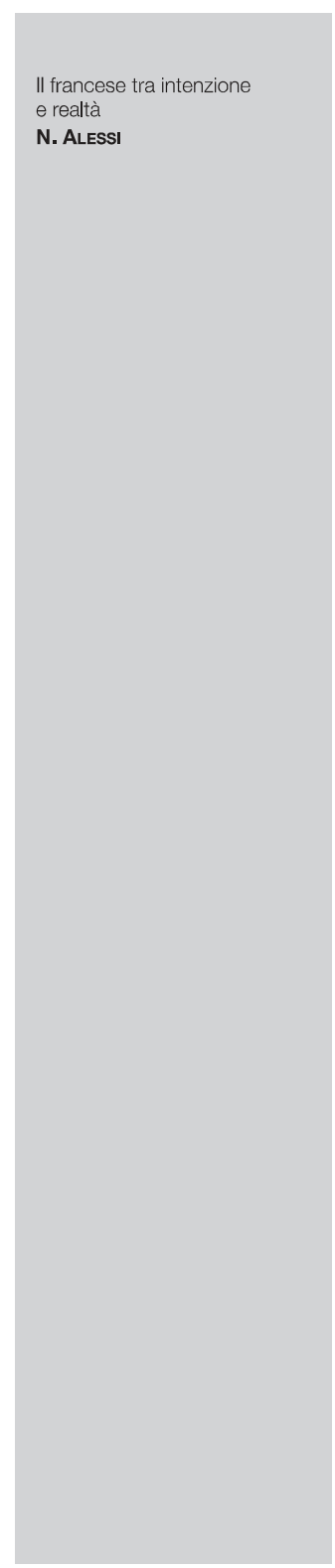

ferma questo quadro. Su un totale di 1688 deliberazioni della Giunta regionale, 75 , ossia circa il $4,5 \%$, sono state redatte in francese. Quasi la metà di queste $(47 \%)$ consiste in sovvenzioni e finanziamenti a iniziative e associazioni culturali valdostane di vario genere; la restante parte ha ad oggetto nomine $(11 \%)$, convenzioni e collaborazioni con enti, accademie e università francesi $(11 \%)$, iniziative di cooperazione transfrontaliera Italia - Francia $(9 \%)$, acquisto di volumi $(8 \%)$, iniziative in favore della lingua francese e del dialetto francoprovenzale $(7 \%)$, toponimi $(2,5 \%)$, disposizioni in materia di archivi $(2,5 \%)$, alpeggi $(1 \%)$ e piano di gestione di Finaosta S.p.A. (1\%).

Risulta dunque evidente la settorializzazione dell'utilizzo della lingua francese nell' ambito dell' attività istituzionale.

Questo atteggiamento politico e istituzionale incide sulle percezioni della popolazione, che mostra, nel tempo, distanza e disaffezione nei confronti della questione linguistica. Agli occhi della generalità il francese rappresenta un patrimonio regionale da rispettare, ma solo fino a quando non diventi troppo ingombrante (si pensi alle proteste per l'esame di Stato), un codice da usare per dimostrare di essere parte di una comunità (in contrapposizione alle altre), ovvero un "titolo" da esibire per la fruizione di particolari privilegi.

Gi si riferisce principalmente alla cosiddetta indennità di bilinguismo, nata come misura rivolta ai dipendenti delle amministrazioni nazionali prestanti servizio in territorio valdostano e successivamente estesa a tutto il comparto unico regionale. Questa scelta del legislatore regionale cela un atteggiamento che pare voler attribuire un particolare status privilegiato alla popolazione valdostana in ragione delle sue peculiarità linguistiche, "premiando» il bilinguismo e mostrando tutte le contraddizioni dell'approccio politico alla questione.

Un ultimo dato che denota la discrasia tra quanto previsto nell'ordinamento regionale e la realtà è la posizione giuridica del francoprovenzale, idioma diffusamente parlato e sostanzialmente poco tutelato. Le previsioni di tutela del patois si limitano alla promozione di attività culturali o extrascolastiche, non riconoscendo a questo idioma la medesima dignità del francese e non prevedendo strumenti per un suo ingresso in pianta stabile nella didattica valdostana. Ciò anche disallineandosi dalle previsioni nazionali contenute nella 1. 15 dicembre 1999, n. 482, che dispone che la lingua francoprovenzale possa essere insegnata nelle scuole dell'infanzia, primarie e secondarie di primo grado dei comuni dove insiste la popolazione della minoranza (che in Valle d'Aosta coin- 


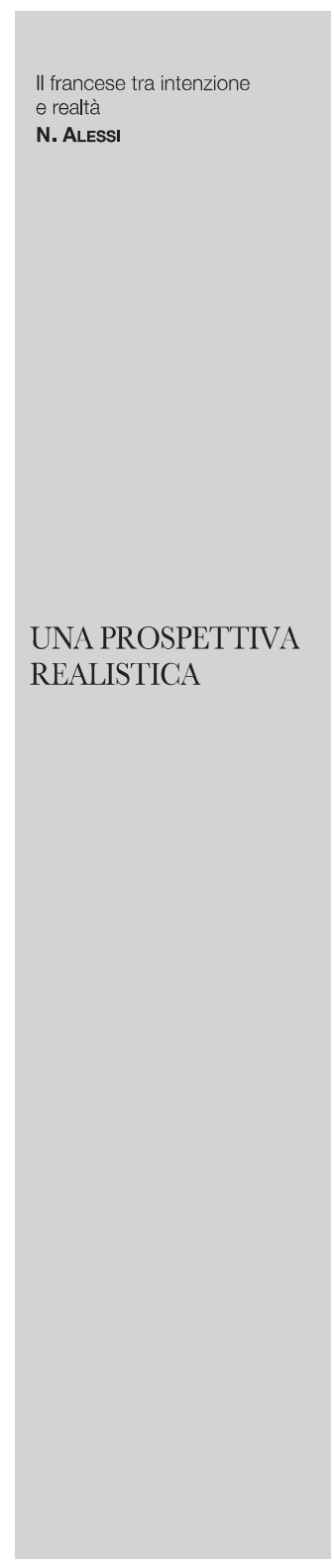

cide con la popolazione di 71 dei 74 Comuni). È incontestabile la forte subalternità del patois a livello formale, quando, al contrario, a livello di pratica esso si dimostra vivo e presente, ufficiosamente utilizzato in qualsivoglia contesto anche istituzionale (riunioni di Giunta regionale ad esempio). A differenza del francese, il patois non gode di un riconoscimento e una legittimazione forti, non potendo così esprimere appieno le proprie potenzialità «espansive» (per esempio nell'attività politica, nelle relazioni con la pubblica amministrazione, oltre che nella scuola). Si realizza così un paradosso: una Regione nata anche per ragioni di peculiarità linguistiche non presenta al suo interno l'atteggiamento di apertura e promozione che lo Stato ha avuto con essa.

In definitiva, calando la normativa statutaria nella realtà, è possibile scorgere l'unicità del modello linguistico valdostano, che mostra un grande scollamento tra previsioni normative, realtà concreta e percezioni.

Un tale scollamento induce a mettere in discussione le ragioni stesse dell'ordinamento linguistico valdostano. Queste considerazioni conducono in effetti a porsi un primo interrogativo di fondo, solo in apparenza banale: ha ancora una ragion d'essere, oggi, un ordinamento regionale strutturato secondo il principio del bilinguismo, con tutte le conseguenze che ne derivano (strutturazione bilingue delle istituzioni, della pubblica amministrazione, prove di francese per i dipendenti pubblici, spazi del francese nelle emittenti, scuola bilingue, ecc.)?

Per fornire delle risposte adeguate occorre utilizzare un approccio integrato: storico-giuridico, ma anche linguistico. Ciò in modo da poter analizzare compiutamente gli aspetti storico-costituzionali della questione senza perdere di vista la realtà della situazione sociolinguistica odierna.

Muovendo da questo approccio, lo sbilanciamento in favore di una risposta affermativa deriva dalla convinzione che, sebbene quasi in disuso, il francese rappresenti comunque un patrimonio in sé di indubitabile valore. In questo senso, il mantenimento di un ordinamento connotato linguisticamente risponde legittimamente alla volontà di tutelare la diversità di un patrimonio culturale, evitando la sua sparizione. In generale, tutelare la diversità linguistica - quando non intesa come «difesa» tout court (in senso escludente) - significa evitare la completa omologazione della società a un modello economico e culturale predominante. La lingua francese nel contesto valdostano è fattore di diversità (della comunità valdostana), che può essere ritenuto un valore insito nel 


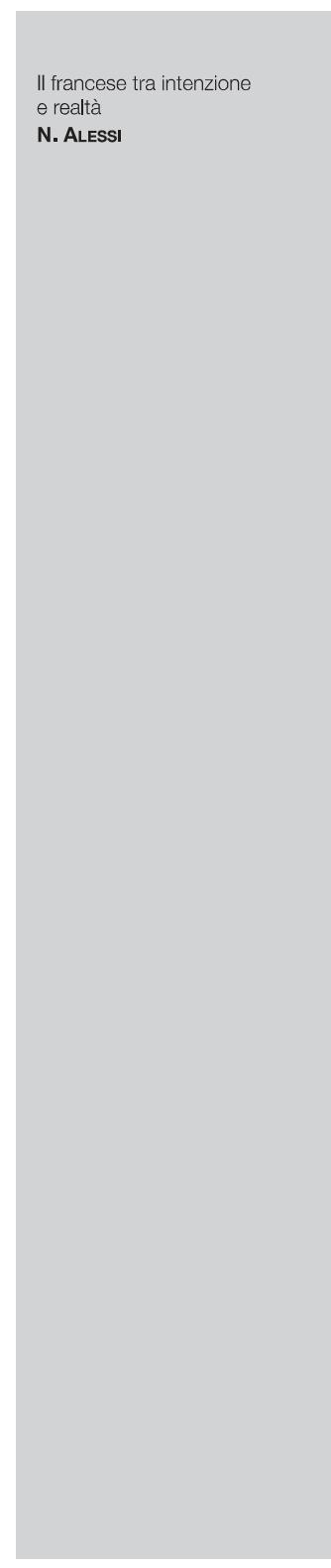

pluralismo della Carta costituzionale italiana. La diversità costituisce dunque un valore culturale e costituzionale che, se tutelato in sé attraverso la protezione dei suoi fattori (come la lingua), e non in un'ottica assiologica, non può non essere condiviso e promosso, perché elemento decisivo nella costruzione dell'identità degli esseri umani e del loro libero sviluppo.

Questa prima considerazione conduce a ritenere che un ordinamento che annoveri tra i suoi obiettivi la tutela del francese mantenga una sua ragion d'essere per motivi storico-culturali, in ragione anche del valore costituzionale riconosciuto alla diversità. Possono essere dunque considerate ancora fondate le misure statutaric che strutturano la Regione in senso bilingue perché basate sulla volontà di salvaguardare una cultura attraverso le istituzioni. Del resto, come osservato dallo stesso Franco Calvetti: "La lingua è il veicolo attraverso cui passano i valori della comunità che la parla". In questo senso, la Regione si fa portatrice di questi valori e lo studio della lingua costituisce un modo per condividerli.

Se questo è vero, adottando l'approccio accennato in precedenza, pare comunque necessaria una taratura realistica del bilinguismo valdostano, che tenga conto del dato fattuale. In questo senso, occorre prendere coscienza che 1) l'unica prospettiva di «sopravvivenza» del francese in Valle d'Aosta consiste nel suo studio, nella sua conoscenza e non nella sua pratica; 2) vi sono disposizioni di matrice linguistica che hanno una ragion d'essere solamente in ambiti bilingui in cui entrambe le lingue sono effettivamente parlate.

La prima valutazione deriva direttamente da uno sguardo alla realtà sociolinguistica valdostana e non ha bisogno di altre argomentazioni. La seconda mette in risalto un approccio realistico al bilinguismo statutario, e permette di analizzare la ragionevolezza delle disposizioni di tutela linguistica in riferimento al peculiare contesto valdostano, proprio perché caratterizzato da una sostanziale assenza del francese nella pratica quotidiana. Il riferimento è a quelle norme che dispongono il superamento di una prova di francese come condizione per l'esercizio di talune attività professionali private o all'interno della pubblica amministrazione. Il tema necessita di un approfondimento che non può trovare sede in questo breve saggio; ma trapela la necessità di razionalizzare questo genere di disposizioni a fronte di una realtà sociolinguistica nella quale non si avverte la necessità dell'utilizzo del francese in determinati contesti - in particolare quelli professionali e funzionariali - dove la condivisione di uno stesso linguaggio tecnico rappresenta la base per l'efficacia della comunicazione e quindi 


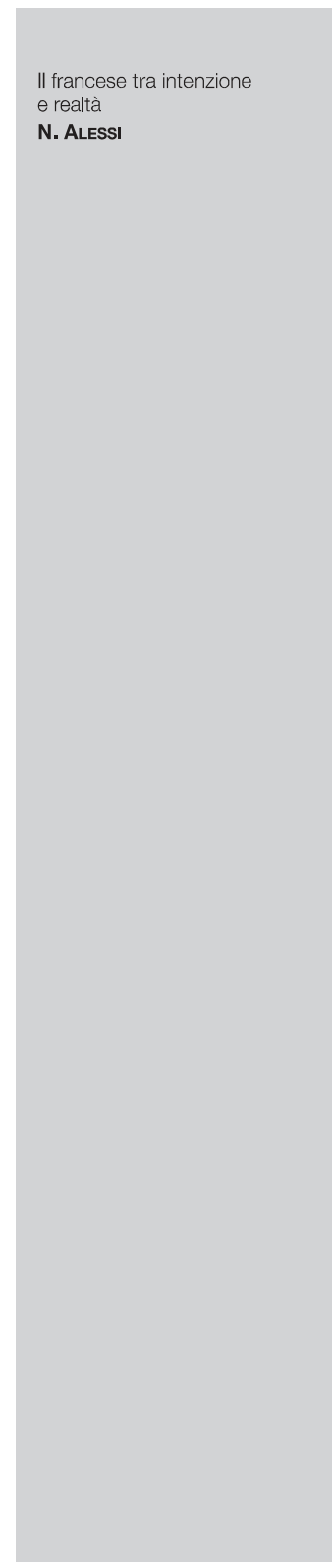

della prestazione. Ciò impone un ripensamento che si farà più delicato in riferimento al personale delle pubbliche amministrazioni, depositarie del bilinguismo statutario. In questo senso, va dato atto che recentemente sono state avanzate proposte di legge per l'abolizione del vincolo del superamento della prova di francese per l'accesso alla dirigenza medica regionale (2), settore ove si rende necessario ricercare un equilibrio tra bilinguismo e esigenze di effettività del servizio pubblico.

Un approccio realistico all'analisi della posizione del francese in Valle d'Aosta conduce anche a prendere coscienza che nei contesti - come quello valdostano - ove allo studio e alla valorizzazione istituzionale della lingua francese non si accompagna la pratica (che involve dinamiche non pienamente gestibili da politiche linguistiche) la forza della storicità della questione linguistica, la sua pregnanza, affievoliscono. Lo scollamento tra norme, realtà e percezioni e la sempre più debole comprensione della giustificazione storica della presenza della lingua francese in Valle d'Aosta possono indurre la popolazione valdostana a avvertire come non ragionevole la sua tutela. In altre parole, la storicità del francese non sembra oggi una ragione sufficiente per giustificare le misure di protezione poste in essere dall'ordinamento valdostano, che rischiano di essere considerate sempre più come imposte, in un contesto caratterizzato dallo scollamento di cui si è detto in precedenza.

Posto il valore che la lingua francese riveste quale fattore di diversità, pare utile indagare se esistano, e quali siano, le prospettive della sua «sopravvivenza». Forse è proprio a partire dal proposto riposizionamento realistico del francese che si possono trarre delle nuove prospettive di vitalità di questa lingua. Invero, prendendo coscienza della distanza tra norma e fatto (3) si potrà attuare quel ripensamento del ruolo del francese che sembra necessario da tempo, anche per rafforzare il suo legame con la comunità che lo studia e lo conosce. Ciò può essere utile anche per comprendere su quali obiettivi potrebbero concentrarsi misure di politica linguistica.

In questo senso, una prospettiva maggiormente realistica induce a concepire lo studio del francese come un'opportunità lasciata dalla tradizione francofona di questo territorio. Questa impostazione si traduce in diversi corollari con differenti prospettive applicative.

In primo luogo, il francese è un'opportunità di creazione di competenze linguistiche utilmente spendibili dentro e fuori del contesto regionale. La promozione di competenze linguistiche nell'otti- 


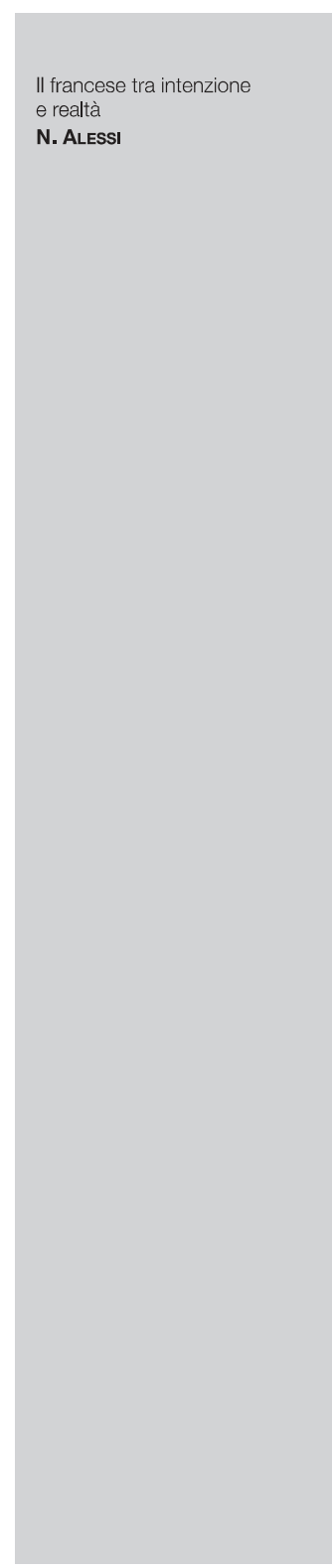

ca della loro spendibilità all'interno e all'esterno della Regione rappresenterebbe una sicura direzione di attualizzazione della questione della lingua in Valle d'Aosta, denotando un superamento dell'autoreferenzialità tipica della retorica identitaria. Anche a livello di percezione, la lingua apparirebbe alla popolazione meno come una costruzione identitaria esclusiva e artefatta, acquisendo interesse quale «prodotto» di una cultura passibile di una valorizzazione in quanto (anche) utile.

Tutto ciò richiede, sul piano delle politiche, che siano, in primis, il giurista e il legislatore a dover acquisire, nei confronti della lingua, un atteggiamento non più difensivo/identitario. In questo senso, nello specifico, andrebbe superata l'idea che indagare sugli aspetti di concreta utilità del francese in termini di competenze svilisca il suo status di lingua ufficiale relegandolo a «lingua straniera».

È riscontrabile un qualche segno di una tale presa di coscienza? La recente legislazione regionale ha fornito degli spunti interessanti in tal senso in ambito scolastico. In effetti, con l'approvazione della l.r. 17 dicembre 2018, n. 11 è stato introdotta una prova di francese inedita nel contesto regionale. Tale prova sarà sostenuta obbligatoriamente a partire dall'a.s. 2019-2020 da parte degli studenti delle classi quinte delle scuole secondarie di secondo grado. Essa condurrà al rilascio di una certificazione linguistica sulla base dei parametri del QCER (B1-C2) da parte della Sovrintendenza agli Studi, che si pone quale ente certificatore delle competenze linguistiche in lingua francese degli studenti valdostani. E questa una grande novità nell'ordinamento regionale, perché per la prima volta viene predisposta una certificazione di lingua che abbia un valore anche all' esterno della Regione.

In secondo luogo il francese è un'opportunità di apertura culturale in due direzioni: dall'interno verso l'esterno e dall'interno nei confronti dell'esterno. In rapporto al primo profilo, il francese si rivela come un'opportunità in quanto la conoscenza di una lingua altra costituisce un "allenamento» alla diversità, che predispone all'apertura culturale. In questo senso, il francese può essere considerato come fattore di educazione all'interculturalità, alla diversità e al dialogo, che favorisce la creazione di reti oltre i confini nazionali. La promozione del francese troverebbe un significato dunque come un'opportunità educativa lasciata dal passato. Nel concreto, concepire il francese in questo modo implica dare una volta di più forza e significato al suo studio a scuola oggi, e soprattutto al valore di una scuola effettivamente bi-plurilingue; ma potrebbe anche essere un impulso per nuove politiche di rafforza- 


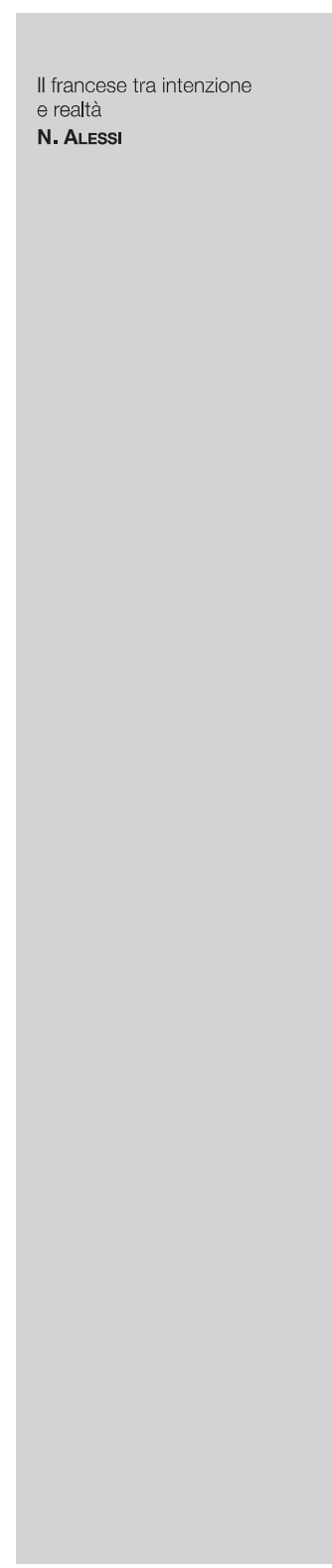

mento del dialogo con Paesi e zone francofone del mondo. Va, del resto, sottolineato, che l'impegno regionale in quest'ultima direzione è oggi modesto. Un interessante riscontro che sembra denotare un approccio istituzionale in tal senso è il sostegno regionale a iniziative di simulazioni parlamentari francofone in Valle d'Aosta e all'estero.

Per quanto riguarda il secondo profilo, il francese rappresenta un'opportunità di apertura della Regione nei confronti dell'esterno in quanto contesto, seppur sui generis, caratterizzato dalla presenza di una lingua praticata in vaste zone del mondo. Il riferimento va necessariamente, in questo periodo storico caratterizzato da fenomeni migratori strutturali, anche a tutti quei Pacsi di emigrazione nei quali il francese è prima o seconda lingua. Come risaputo, una delle principali esigenze delle cosiddette nuove minoranze non è quella di vedere riconosciuto loro il diritto di parlare la propria lingua, quanto piuttosto di riuscire a comunicare nella lingua del luogo ove si trovano per potersi proficuamente inserire e integrare nel contesto di destinazione. In questo senso, la lingua potrebbe costituire un fattore di facilitazione per l'integrazione di migranti francofoni in Valle d'Aosta, gettando le basi per un modello di accoglienza fondato, quando possibile, sulla compatibilità linguistica del contesto di arrivo. Sfruttare le peculiarità linguistiche della Regione per finalità di integrazione - con tutti gli aspetti virtuosi che reca con sé tale processo soprattutto in un contesto caratterizzato dallo spopolamento dei paesi di montagna - potrebbe essere la nuova sfida di una piccola Regione diversamente bilingue. 


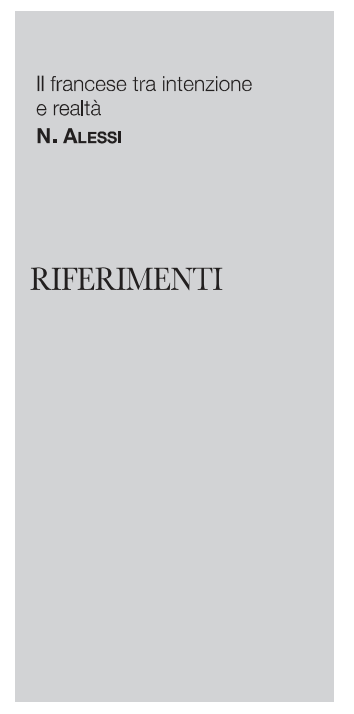

BERRUTO G. 2003. Una Valle d'Aosta, tante Valli d'Aosta? Considerazioni sulle dimensioni del plurilinguismo in una comunità regionale, in Fondation Emile Chanoux, Une Vallée d'Aoste bilingue dans une Europe plurilingue - Una Valle d'Aosta bilingue in un'Europa plurilingue, Tipografia Valdostana, Aosta.

MASTROPAOLO A., POGGI A. 2017. La specialità valdostana tra fatto e norma, Le Regioni, n. 6: 1165-1174.

PALICI DI SUNI C. E. 2002. Intorno alle minoranze, Giappichelli, Torino;

\section{NOTE}

(1) Gaetano Berruto (2003: 44-53), definisce il contesto valdostano come caratterizzato da un bilinguismo sociale endocomunitario con dilalìa multipla; questa definizione dà atto della complessità della situazione valdostana, che fuoriesce dai rapporti tra la lingua francese, quella italiana, il dialetto francoprovenzale e, più marginalmente, il dialetto piemontese; essa si sostanzia nella presenza di due lingue «alte» (italiano e francese), caratterizzate da un rapporto non paritario (l'italiano erode gli spazi del francese), e di due varianti linguistiche «basse» (francoprovenzale e piemontese), l'una (il francoprovenzale) più diffusamente utilizzata rispetto all'altra.

(2) Ci si riferisce alla proposta di legge n. 13 del 23 novembre 2018, recante «Disposizioni in materia di accertamento della conoscenza della lingua francese per i dirigenti del ruolo sanitario. Modificazioni alla legge regionale 25 gennaio 2000, n. 5»; essa prevede che "in casi eccezionali di carenza di tali figure individuati con deliberazione della Giunta regionale su proposta dell'Azienda USL, l'ammissione allo svolgimento delle prove di esame per l'assunzione a tempo indeterminato (...) presso l'Azienda stessa non è subordinata al superamento della prova di accertamento della conoscenza della lingua francese. Nel caso in cui non sia superata la prova di accertamento linguistico, gli idonei alle restanti prove concorsuali sono inseriti nella graduatoria di merito in coda rispetto ai vincitori e agli idonei che hanno superato con esito positivo tale prova".

(3) Sulla distanza tra norma e fatto nel contesto valdostano, anche se in riferimento, più in generale, allo scollamento tra ordinamento formale e la sua attuazione concreta nella vita istituzionalepolitica (cf. Mastropaolo et al. 2017). 